\title{
A STUDY OF MALARIA IN RELATION TO HIV AND SYPHILIS AMONG PATIENTS VISITING BHERI ZONAL HOSPITAL
}

\section{ABSTRACT}

The study was conducted in patients, who were febrile with signs of anaemia, splenonregaly and the classic cycle of coldness, fever peaks and sweats from January to September 2000 in Bheri Zonal Hospital, Nepal. The study aimed to find out relationship of malaria with HIV and syphilis.

Two hundred and twenty three blood samples collected were processed and observed using the methods of ELISA and RPR.

Among total 223 suspected risk group cases, 6 individuals were detected as HIV positive and 12 individuals had syphilis. Co-infection with malaria \& HIV was detected in 2 patients and malaria with syphilis in 8 patients. Similarly 2 patients were co-infected with malaria, HIV and syphilis. A small positive correlation $(p<0.5)$ of malaria, HIV and Syphilis was detected.

Key Words: Malaria, HIV, Syphilis, ELISA, RPR, Nepal.

\section{INTRODUCTION}

Both malaria and HIV kill millions of people each year and both diseases are scourges of developing nations in Africa, India, South East Asia and South America. But HIV is pandemic, spread from person to person by sexual contact in an increasingly mobile world. Malaria is endemic, dependent on a local symbiosis between infected anopheline mosquitoes and humans. The severe symptoms of malaria caused by the parasite plasmodium falciparum appear within days and bring death to about 15 to $25 \%$ of those stricken when great quantities of infected red blood cells are destroyed in a single burst. HIV infection is a slow, insidious process that can take years to deplete immunologically crucial white blood cells. It results in death for nearly all untreated patients. ${ }^{1}$

The immune system of our body to fight malaria is not fully understood, although it is clear that both humoral and cellmediated immunity are involved and that various T-cell subsets are important for regulating the immune response. HIV too has an intricate relationship with the immune system, and it appears that there may be several points of intersection between the pathogenesis and response to each disease. ${ }^{1}$

Studies are contradictory about the frequency and severity of malaria in HIV infected people. Malaria does not appear to

* Infectious and Tropical Disease Research Centre, Tripureshwor, Kathmandu, Nepal.

** Institute of Medicine, TUTH, Kathmandu, Nepal.

*** Tribhuvan University, Kirtipur, Kathmandu, Nepal.

Address for correspondence : Prakriti Raj Kandel

Foreign Student's Hostel, Room No. B 12, University P.O.

Tha-Ton Road, Kamayut Township, Yangon, MYANMAR

Email: rnem@mptmail.net.mm 
act as a classic opportunist in immune compromised hosts. People who have grown up in endemic regions often retain partial immunity to malaria and there is no solid evidence that this immunity is lost as HIV disease progress. ${ }^{1}$

HIV infection causes increasing cellular immunosuppression and could thus result in an impaired immune response to malaria leading to failure to prevent infection or suppress parasitaemia and clinical disease. ${ }^{2} \mathrm{HIV}$ infection is associated with lower $\mathrm{CD} 4 \%$ and higher neopterin and beta 2 micro globulin levels and co-infection with syphilis is associated with a further lowering of $\mathrm{CD} 4 \%$, suggesting a greater suppression of immune system. Co-infection with malaria is associated with a modest immune disturbance. ${ }^{3}$

In the light of these issues, present study aims to state and analyse the relationship of malaria with HIV and Syphilis.

\section{METHODOLOGY}

Blood sample collection was carried out between January to September, 2000 from the patients with signs of anaemia, enlargement of the spleen and the classic cycle of coldness, fever peaks and sweats attending out patient department and those admitted in the hospital. Venous blood was collected in clean, dry and sterile centrifuge tubes from patient of all ages and both sexes. These tubes, sealed with seal cap were kept in refrigerator before processing of samples.

Blood specimens collected in tubes were centrifuged at 10,000 rpm for 5 minutes at room temperature within 3 hours of storage. The serum was drained into a $0.5 \mathrm{ml}$ Eppendrof tube then, kept at $-200 \mathrm{c}$ until used.

For antibody detection, serology was performed on sample from malaria, HIV and syphilis suspected patients. Enzyme-linked immunosorbent assay (ELISA) was performed in case of malarial and HIV antibody detection whereas rapid plasma reagin (RPR) test for the detection of the antilipoidal antibodies in syphilis cases.

ELISA was performed using invitro cultured P. falciparum antigen for malarial antibodies detection whereas the Biochem DETECT-HIVTM (Trademark of Biochem Immuno Systems Inc.) test kit was used for detection of antibody to HIV-1 and HIV-2 in human serum or plasma. RPR Card Test/Carbon antigen Kit from Tulip Diagnostics (P) LTD. was used for syphilis testing. The rapid plasma reagin (RPR)/carbon antigen test is a macroscopic non-Treponemal flocculation test for the detection and quantitation of antilipoidal antibodies.

\section{RESULTS}

Out of 223 patients tested for HIV and Syphilis, 6 (2.7\%) were HIV positive and 12 (5.4\%) were RPR positive. Between these HIV and Syphilis positive patients, 2 (1\%) cases were coinfected with HIV, Malaria and Syphilis. Similarly 8 (3.6\%) cases were co-infected with Syphilis and Malaria; 2 (1\%) cases were co-infected with HIV and Malaria. Only HIV infected patient without any co-infection with malaria or syphilis were 2 (1\%). Also only Syphilis infected patient were $2(1 \%)$. 